\title{
Evaluation of Combined Anatomic Anterior Cruciate Ligament and Anterolateral Ligament Reconstruction in Anterolateral Rotatory Instability of the Knee

\author{
M.E.Abdelnaby, E.M.Baioumy and B.A.Khalil
} \\ Orthopedics surgery Dept., Faculty of Medicine, Benha Univ., Benha, Egypt \\ E-Mail: B.Khalil @gmail.com
}

\begin{abstract}
Some studies has demonstrated improved dynamic rotational stability with a double bundle reconstruction. However, another multiple studies did not find improved functional rotational stability, also cost and time needed and the increased incidence of cyclops syndrome are causes of technique limitation. We aimed to evaluate of results of combined anatomic ACL and anterolateral ligament reconstruction in patients with anterolateral rotatory instability of the knee. Twenty patients with chronic ACL insufficiency were subjected to combined anatomic single-bundle ACL reconstruction and anterolateral ligament reconstruction by semitendinosus and gracilis tendons. International Knee Documentation Committee (IKDC) subjective knee evaluation form (page 76, 77) was filled and scores were recorded. The results of this technique show excellent (normal) results in 18 patients grade A according to objective IKDC and very good (near normal) in 2 patients. Range of motion at 3 months follow up was limited to $90^{\circ}$ to $110^{\circ}$ in 7 patients, all these patients were improved with physiotherapy and rehabilitation programs with no need to other management. Isometric ALL is highly important to prevent tight lateral compartment of the knee and prevent limited range of motion and lateral side knee pain. There is no major complication associated with this technique to limit its use.
\end{abstract}

\section{Introduction}

The main complaint of a patient with ACL tear after an injury or failed reconstruction is instability in particular, these patients complain of rotational instability with pivoting or cutting activities [1].

The pivot-shift test, which evaluates both anterior tibial translation and axial tibial rotation, is considered the most specific examination to evaluate rotational knee stability and knee function after ACL reconstruction [2,3].

The presence of high degree positive pivot-shift test result is predictive of a failure to return to the previous level of activity, and poor subjective outcome scores after ACL reconstruction [4].

Intra-articular ACL reconstruction is associated with superior quality of life; sports function and knee symptoms when compared to non-operative treatment [5].

As the incidence of ACL reconstruction (ACLR) has a significant increase over the last two decades, the revision rates for this operation has increased also due to many causes as graft rupture in young patients participating in contact, pivoting sports, low rates of return to pre-injury levels of sport and persistent rotatory instability, which remain important post-operative clinical issues and represent a significant surgical burden [6-11].

Isolated rupture of the ACL causes small increase of internal rotation, so an obvious increase of internal rotation laxity implies damage to the lateral extra articular structures resulting in persistent anterolateral rotational knee instability $[12,13]$.

Isolated intra-articular ACL reconstruction may be relatively not enough for controlling internal rotation. Increasing the tension in an isolated ACL graft may lead to over-constraint of anterior translation laxity, yet allow residual rotational abnormality to persist [18]. With some having a residual pivot shift, and increased tibial internal rotation in gait, and in squatting $[15,16]$.

Interest has been renewed in lateral extra-articular reconstruction in association with ACLR in the primary setting as a method for reducing the rate of graft failure and thought to mechanically act on the lateral periphery of the joint to provide an increased lever arm for controlling rotation (due to its greater distance from the center of rotation of the knee) than an isolated intra-articular reconstruction and to prevent subluxation of the tibial plateau and hence the knee become more stable [17].

The aim of this study was evaluation of results of combined anatomic ACL and anterolateral ligament reconstruction in patients with anterolateral rotatory instability of the knee.

\section{Patient and method}

Twenty patients with chronic ACL insufficiency were subjected to combined anatomic single-bundle ACL reconstruction and anterolateral ligament reconstruction by semitendinosus and gracilis tendons. The period of the study was from June 2018 to March 2020. All surgeries were done by the same surgeon with the same technique that will be described later. All surgeries were done in Benha University hospital and Elmogamma elteby hospital in Tanta. The criteria of the patients included in this study were;

\section{Inclusion criteria}

- Patients with ACL insufficiency.

- Moderate to severe rotatory instability as revealed by a pivot-shift test grade 2 or 3 (on a laxity scale ranging from score $0=$ negative, $1=$ glide, $2=$ clunk, to $3=$ subluxation) and lachman sign with soft end point.

- A minimum interval of 4 weeks between trauma and surgery.

- Age less than 40 years.

\section{Exclusion criteria}

- Acute injuries (less than 4 weeks).

- Skeletally immature patients.

- Previous surgical procedures on the same or the contra lateral knee.

- Other concomitant ligamentous injuries of the knee. 
- Preoperative radiological signs of knee arthritis.

- Imaging evidence of severe chondral damage on both the patellar surface and medial or lateral femoral condyles.

- Malalignment of the knee as varus or valgus knees.

The presence of concomitant minor medial or lateral meniscal lesions was not considered to be an exclusion criterion.

\section{Preoperative examination \\ All patients were subjected to}

- History taking.

- Full general examination.

- Local examination of injured knee for range of motion, stability tests (anterior drawer, lachman and pivot shift) comparing it with the other uninjured contralateral knee.

- International Knee Documentation Committee (IKDC) subjective knee evaluation form was filled and scores were recorded.

- Plain X-ray of both knees in standing anteroposterior, lateral and sunrise views.

- Magnetic resonance imaging of the injured knee.

The rehabilitation after combined ALL reconstruction and ACL reconstruction, was carried out in a similar way to conventional ACL rehabilitation. Early post-operative focus on control of inflammatory signs, pain and effusion, initial quadriceps awakening with both voluntary and electro stimulated muscle contraction, and emphasis was placed on achieving immediate full extension to reduce the risk of bleeding and adhesion or cyclops formation.

Passive flexion and patellar mobilization, avoiding eccentric quadriceps contraction, was also performed. The patient was discharged on the same day or on the day after surgery, without immobilization. The patients were instructed for total weight-bearing (as tolerated) with the aid of crutches, hamstring stretching to prevent flexion attitude, later gait training, active range of motion and progressive muscle strengthening till reaching full activity. Every patient was checked weekly in the $1^{\text {st }}$ month for wound evaluation, knee swelling, signs of infection, and progression of rehabilitation instructions.

Every patient was evaluated three and six months postoperatively stability tests (anterior drawer, lachman and pivot shift) comparing it with the preoperative records.

International Knee Documentation Committee (IKDC) subjective knee evaluation form was filled by the every patient and scores were recorded and compared with the preoperative records.

\section{Results}

All twenty patients included in this study were males with the youngest patient at surgery was 18 years old and the oldest one was 36 years old with mean age 23.65 years \pm 5.18 standard deviation (SD) (table. 1$)$. The side affected was left in thirteen patients (65\%), and right side in seven patients $35 \%$ table 2 . The mode of trauma was non-contact trauma in seventeen patients $(85 \%)$ and direct trauma in three patients $(15 \%)$.
The shortest period between the trauma and operation was 2 months and the longest period was 25 months, with the mean period was 14 months $\pm 11.5 \mathrm{SD}$

The mean follow up period was 12.75 month with the minimum follow up was 9 months and the maximum follow up period was 19 months \pm 3.5 SD . Preoperative all patients were showing no limitation of ROM in comparison to the normal knee.

At three months postoperative there was 7 patients (35\%) with limited ROM 90-110 knee flexion. At six months postoperative all patient regain full ROM regarding to the contralateral knee.

All twenty patients show negative varus and valgus stress tests pre and post-operative. the preoperative results of anterior drawer test show 17 patients $(85 \%)$ grade 3 and 3 patients $(15 \%)$.

Lachman test preoperative was grade 2 in 8 patients $(40 \%)$ and grade 3 in 12 patients $(60 \%)$. Pivot shift test results was grade 2 in 13 patients $(65 \%)$ and grade 3 in 7 patients $(35 \%)$.

6 months post-operative the results of stability tests were no patients grade 2 nor 3 , but 5 patients $(25 \%)$ were grade 1 anterior drawer, 3 patients $(15 \%)$ grade 1 lachman test and 1 patient $(5 \%)$ grade 1 pivot shift test

Symptoms take 37 point in the IKDC score and the results preoperative were recorded in all twenty patients with the maximum score was 21 , and the minimum score was 16 with the mean score of symptoms was $18.85 \pm 1.53$ SD

The recorded post-operative symptoms show maximum value 35 , minimum value 29 and the mean result $32.4 \pm$ 1.73 SD $(\mathrm{P}<0.0001)$

The total sum of all IKDC items is 87 points, and the preoperative results show the maximum sum was 58 which mean IKDC score was 66.66 , the minimum total sum was 42 which mean IKDC score was 48.27 and the mean preoperative sum was $49.45 \pm 4.42 \mathrm{SD}$, this mean IKDC score $56.83 \pm 5.08 \mathrm{SD}$.

Maximum Postoperative total sum was 83 which correspond to 95.4 IKDC score, minimum postoperative total sum was 68 which correspond to 78.16 IKDC score, and the mean post-operative total sum was $76.55 \pm 4.27$ SD which correspond to $88.07 \pm 5.03$ as the mean IKDC score postoperative $(\mathrm{P}<0.0001)$. Table .8 and figure. 48 show the results of total sum and IKDC score pre and postoperative.

Knee function take 10 points in IKDC score. Preoperative results were 6 as the maximum value, 4 as the minimum and the mean was $4.85 \pm 0.67$ SD . Postoperative knee function results show 10 as the maximum value, 7 as the minimum and the mean was $8.85 \pm 0.88 \mathrm{SD}$ $(\mathrm{P}<0.0001)$.

Sport activity take 40 points in the IKDC score and preoperative results show maximum score 31 , minimum score was 22 with the mean $25.8 \pm 2.63$ SD Post-operative results were 38 as maximum value, 31 as minimum value and the mean $35.25 \pm 1.92(\mathrm{P}<0.0001)$.

According to the objective IKDC form, the preoperative results abnormal (grade $\mathrm{C}$ in 7 patents and grade D in 13 patients). Three months postoperative the 
objective IKDC score change to normal or nearly normal (grade $\mathrm{A}$ in 11 patients and grade $\mathrm{B}$ in 9 patients). 6 months post-operative the 18 patients were grade $\mathrm{A}$ and 2 patients were grade $\mathrm{B}(\mathrm{P}<0.0001)$, table 9 show all results Table (1).

There was 6 patients $(30 \%)$ with small meniscal tears, all treated with partial menisectomy and debridement with no total menisectomy nor meniscal repair was done for any patients included in this study.

Postoperative calf hematoma was occurred in 2 patient (10\%), and postoperative thigh hematoma was occurred in 4 patients $(20 \%)$, all 6 patients were treated with conservative measures (fomentation, elevation, anti- edematous medications), resolution occur in 3 to 5 weeks Fig (1).

Superficial wound infection in the graft harvesting site was occurred in 1 patient $(5 \%)$ and was treated with short course of antibiotic and dressing.

Lateral knee side pain in 5 patients $(25 \%)$ in early follow up periods which improved gradually in second visits. Anterior knee numbness and paresthesia was occurred in 3 patients causing no limitation in rehabilitation program nor functional activity of the knee.

Range of motion at 3 months follow up was limited to $90^{\circ}$ to $110^{\circ}$ in 7 patients, all these patients were improved with physiotherapy and rehabilitation programs with no need to other management.

Table (1) Objective IKDC scores preoperative, 3 and 6 months.

\begin{tabular}{lcccc}
\hline Objective IKDC & Pre-operative & 3 months post-operative & 6 months Post-operative & p \\
\hline A & 0 & 11 & 18 & $<0.0001$ \\
B & 0 & 9 & 2 & \\
C & 7 & 0 & 0 & \\
D & 13 & 0 & 0 & \\
\hline
\end{tabular}

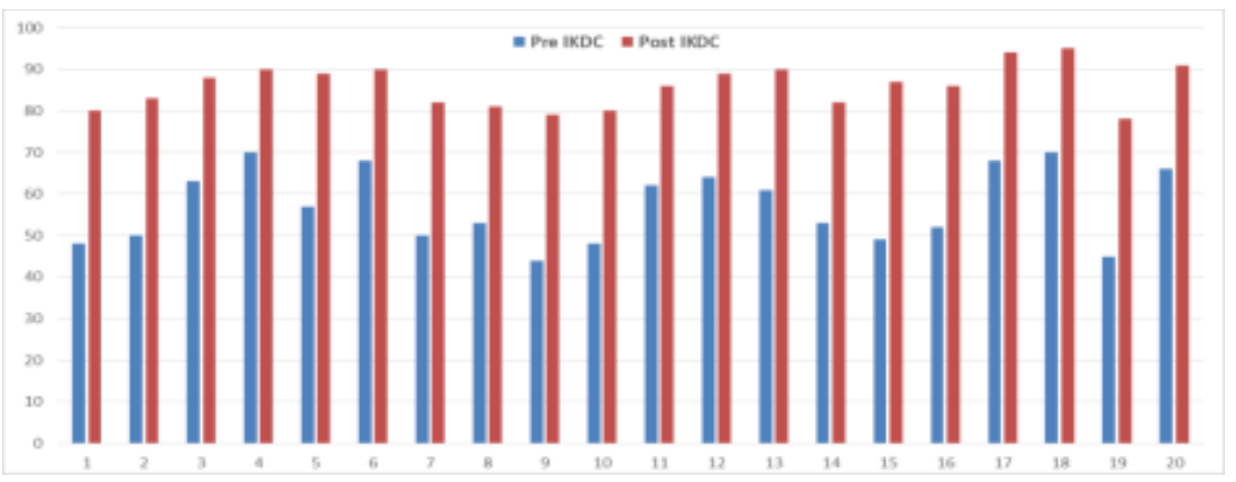

Fig (1) Preoperative and postoperative IKDC score results.

\section{Discussion}

During the past few decades, ACL reconstruction has significantly advanced. Surgeons and scientists are working hard on improving the functional outcomes and quality of life of patients with ACL injuries. Excellent results were obtained with such development. ACL reconstruction is an effective surgery with $75 \%$ to $90 \%$ of patients reporting good or excellent outcomes; however, varying degrees of rotational instability remains an issue in up to $25 \%$ of patients [18].

Failure of ACL reconstruction is a great issue to both orthopaedics surgeons and patients. There is many causes for that failure, tunnel malposition, abnormal mechanical loads as acute traumatic event chronic repetitive movement, inappropriate accelerated rehabilitation postoperatively, and misdiagnosed associated like medial collateral injuries, posterolateral corner Posterior cruciate ligament injuries [19].

A significant number of patients (10\% to $15 \%)$ will require a revision. An ACL reconstruction can be considered a relative failure when the outcomes - as indicated by patient-based outcome measures - do not correspond with the patient's expectations and requirements [20].

One of the common presentation of ACLR failure is recurrent instability which is defined as failure of the reconstructed ligament to provide adequate anterior and/or rotatory stability to the knee. Despite satisfactory clinical results, isolated ACL reconstructions do not restore normal kinematics and biomechanics of the knee, and they particularly do not fully control knee rotational instability. A positive pivot shift test of varying grades can persist in $15 \%$ to $30 \%$ of patients after ACL reconstruction [21].

An anatomical ACL reconstruction using the double bundle reconstruction technique has been advocated to improve rotational stability. Some studies has demonstrated improved dynamic rotational stability with a double bundle reconstruction. However, another multiple studies did not find improved functional rotational stability, also cost and time needed and the increased incidence of cyclops syndrome are causes of technique limitation [22].

Biomechanical studies was performed to evaluate the anterior translation and rotational instabilities after ACL tear and its secondary restraints. The results showed that 
selective cutting of the ACL posterolateral bundle alone did not show any significant increase in anterior tibial translation or internal rotation at different angles of knee flexion [23].

The additional lesion to the anteromedial bundle produced a noticeable increase in anterior translation compared with isolated lesion to the posterolateral bundle, but with an insignificant increase in internal rotation. The addition of an anterolateral structure lesion with a completely cut ACL resulted in a significant increase in both anterior tibial translation and internal rotation, in addition, other studies showed that injuries to the anterolateral ligament of the knee produce a positive pivotshift sign in knees with intact ACL [24].

In a case series of patients with combined ACL and ALL reconstruction, significant improvements were observed in objective and subjective outcomes at a mean follow-up of 32.4 months. Our findings in this study are very similar to what was found in that study. None of our patients nor their patients had a pivot shift of grade 2 or 3 and an IKDC score of C or D at final follow-up [25,26].

Thaunat et al [27] study is the largest published series of any type of lateral extra-articular procedures and the only previously published study that specifically focuses on reoperation rates and complications. Thaunat et al reported that the combined procedure was associated with a very low rate of specific complications $(0.5 \%)$ and a reoperation rate of $13.1 \%$. Thaunat et al highlighted that in large series of isolated ACLRs with similar durations of follow-up, reported rates of reoperation have varied between $18.9 \%$ and $26.7 \%$

Based on previous there was an expected trend toward a lower graft rupture rate with combined ACL ALLR (0.9\%) compared with isolated ACLR with BPTB auto graft $(5.4 \%)$ and significantly improved subjective IKDC, Lysholm, and KOOS (Quality of Life and Activities of Daily Living subscale) scores [28].

It is interesting to note that the rate of reoperation for cyclops lesions was significantly higher in the isolated ACLR group. Numerous reports, including meta-analyses, indicate that ACLR with a BPTB graft is associated with a significantly higher risk of extension deficit [29].

Previous non-anatomic extra-articular techniques, despite having good rotational control, are causing donor site morbidity, cosmetic problems, stiffness, limited ROM, patellofemoral crepitation, poor subjective and clinical results, and degenerative changes in the lateral compartment due to placement of excessive pressure on the lateral compartment. These poor results could now be explained by a combination of imperfectly anatomic ACL reconstruction, an empirical (non anatomic) extra-articular lateral tenodesis, and a postoperative protocol involving immobilization of the knee [30].

The significant difference in this technique compared with previously published extra-articular lateral tenodesis is positioning of the ALL femoral tunnel and securing the graft in full extension and neutral rotation [31].

Possible risks of this technique include anterolateral tibial fracture or tunnel collision if the tunnels are not performed properly, tight lateral compartment of the knee if isometery of the ALL is not followed, also ACL graft diameter may be small although we have never experienced this issue. Iliotibial band irritation due to the small access for femoral screw insertion. One limitation would be a gracilis graft that is not long enough for extraarticular reconstruction, although we have never see this problem.

Although our study is a prospective study, there are some limitations of this study, the follow-up is limited to around 12 months. Longer follow-up is necessary for better evaluation of the benefits and the potential long term complications of this surgical procedure. A comparative study may be useful in evaluating the results of this surgical procedure with respect to either single or doublebundle isolated anatomic ACL reconstruction or other extra articular augmentation techniques. Radiological changes in the lateral compartment of the knee need to be evaluated and correlated with the patient's clinical condition in long term follow up.

\section{References}

[1] C.M. Hettrich, W.R. Dunn, E.K. Reinke, The rate of subsequent surgery and predictors after anterior cruciate ligament reconstruction: two- and 6-year follow-up results from a multicenter cohort. Am J Sports Med ,Vol.41(7), PP.1534-1540,2013.

[2] F.H. Fu, Double-bundle ACL reconstruction. Orthopedics, Vol. 4(4), PP.281-283,2011.

[3] M.S. Kocher, J.R. Steadman, K.K. Briggs, Relationships between objective assessment of ligament stability and subjective assessment of symptoms and function after anterior cruciate ligament reconstruction. Am J Sports Med.Vol. Vol.32(3), PP.629-634. 2004.

[4] S. Kubo, H. Muratsu, S. Yoshiya Reliability and usefulness of a new in vivo measurement system of the pivot shift. Clin Orthop Relat Res, Vol. 454, PP.5458. 2007.

[5] C.L. Ardern, S. Sonesson, M. Forssblad, Comparison of patient-reported outcomes among those who chose ACL reconstruction or non-surgical treatment. Scand J Med Sci Sports,Vol. 27 (5), PP.535-544. 2016.

[6] G.V. Kamath, T. Murphy, R.A. Creighton, Anterior cruciate ligament injury, return to play, and reinjury in the elite collegiate athlete: analysis of an NCAA Division I Cohort. Am J Sports Med,Vol. 42, PP. 1638-1643. 2014.

[7] M.J. Feucht, M. Zens, The anterolateral ligament of the knee: anatomy, biomechanics, and clinical implications. CurrOrthop Pract,Vol. 27, PP.247-253. 2016.

[8] S. Zaffagnini, F. Urrizola, (Residual rotatory laxity after anterior cruciate ligament reconstruction: how do we diagnose it? Curr Orthop Pract, Vol. 27, PP.241246. 2016.

[9] A. Persson, A.B. Kjellsen, K. Fjeldsgaard Registry data highlight increased revision rates for Endobutton/ Biosure HA in ACL reconstruction with hamstring tendon autograft: a nationwide cohort study from the 
Norwegian Knee Ligament Registry, 2004-2013. Am J Sports Med,Vol. 43(9), PP.2182-2188. 2015.

[10]L. Van der Watt, M. Khan, B.B. Rothrauff, The structure and function of the anterolateral ligament of the knee: a systematic review. Arthroscopy,Vol. 31(3), PP.569-582 e563. 2015.

[11]D. Wasserstein, A. Khoshbin, T. Dwyer, Risk factors for recurrent anterior cruciate ligament reconstruction: a population study in Ontario, Canada, with 5-year follow-up. Am J Sports Med,Vol. 41(9), PP.20992107. 2013.

[12]A. Andrew. Anterolateral knee biomechanics Knee Surg Sports Traumatol Arthrosc.Vol. 25, PP.10151023. 2017.

[13]D.T.T. Lie, A.M.J. Bull, A.A. AmisPersistence of the mini pivot shift after anatomically placed anterior cruciate ligament reconstruction. Clin Orthop Relat Res, Vol.457,PP.:203-209, 2007.

[14] P.B. Lewis, A.D. Parameswaran, J.P. Rue, Systematic review of single-bundle anterior cruciate ligament reconstruction outcomes: a baseline assessment for consideration of double-bundle techniques. Am J Sports Med,Vol. 36, PP.2028-2036, 2008.

[15]A.D. Georgoulis, S. Ristanis, V. ChouliarasTibial rotation is not restored after ACL reconstructionwith a hamstring graft. Clin Orthop Relat Res,Vol. 454, PP.89-94, 2007.

[16] T. Zantop, T. Schumacher, S. Schanz, Double-bundle reconstruction cannot restore intact knee kinematics in the ACL/LCL-deficient knee. Arch Orthop Trauma Surg,Vol.130, PP.1019-1026, 2010.

[17] M. Brian. Devitt The role of lateral extra-articular tenodesis in primary anterior Cruciate ligament reconstruction A systematic review with meta-analysis and best-evidence synthesis. Orthop J Sports Med. Oct, Vol.5(10), 2017.

[18] A.F. Anderson, J.r. Snyder RB, A.B. Lipscomb Anterior cruciate ligament reconstruction: a prospective randomized study of three surgical methods. Am J Sports Med, Vol.29, PP. 272-279, 2001.

[19] G.Y. Song, L. Hong, H. Zhang Clinical outcomes of combined lateral extra-articular tenodesis and intraarticular anterior cruciate ligament reconstruction in addressing high-grade pivot shift phenomenon. Arthroscopy,Vol. 32(5), PP.898-905, 2016.

[20]J.r. Bach BR Revision ACL reconstruction: indications and technique. Textbook of Arthroscopy. Philadelphia: Elsevier, PP. 896, 2004.
[21] S. Ristanis, N. Stergiou, K. Patras, Excessive tibial rotation during high-demand activities is not restored by anterior cruciate ligament reconstruction. Arthroscopy,Vol.21(11), PP.1323-1329, 2005.

[22] K. Yasuda, E. Kondo, H. Ichiyama, Anatomic reconstruction of the anteromedial and posterolateral bundles of the anterior cruciate ligament using hamstring tendon grafts. Arthroscopy,Vol. 20(10), PP.1015-1025, 2004.

[23] M. Tanaka, D. Vyas, G. Moloney What does it take to have a high-grade pivot shift?. Knee Surg Sports Traumatol Arthrosc,Vol. 20, PP.737- 742, 2012.

[24] M. Odensten, J. Gillquist Functional anatomy of the anterior cruciate ligament and a rationale for reconstruction. J Bone Joint Surg Am,Vol. 67, PP.257-262. 1985.

[25] L.A. Norwood, J.R Andrews, R.C Meisterling, Acute anterolateral rotatory instability of the knee. J Bone Joint Surg Am,Vol. 61, PP.704-709, 1979.

[26] A. Ferretti, E. Monaco, A. Ponzo, Combined intraarticular and extra-articular reconstruction in anterior cruciate ligament-deficient knee: 25 years later. Arthroscopy,Vol. 32(10), PP.2039-2047, 2016.

[27] M. Thaunat, G. Clowez, A. Saithna, Reoperation rates after combined anterior cruciate ligament and anterolateral ligament reconstruction: a series of 548 patients from the SANTI study group witha minimum follow-up of 2 years. Am J Sports Med. Vol.45(11), PP.2569-2577, 2017.

[28] M. Marcacci, S. Zaffagnini, G. Giordano Anterior cruciate ligament reconstruction associated with extraarticular tenodesis: a prospective clinical and radiographic evaluation with 10- to 13-year follow-up. Am J Sports Med,Vol. 37(4),PP.707-714, 2009.

[29] M. Ahlde' n, J. Kartus, L. Ejerhed, Knee laxity measurements after anterior cruciate ligament reconstruction, using either bone-patellar-tendon-bone or hamstring tendon autografts, with special emphasis on comparison over time. Knee Surg Sports Traumatol Arthrosc. Vol.17(9),PP.1117-1124.2009.

[30] B.J. Moyen, J.Y. Jenny, A.H. Mandrino, Comparison of reconstruction of the anterior cruciate ligament with and without a Kennedy ligament-augmentation device: a randomized, prospective study. J Bone Joint Surg Am,Vol. 74(9), PP.1313-1319,1992.

[31]B. Sonnery-Cottet, M. Thaunat, B. Freychet, Outcome of a combined ACL and ALL reconstruction technique with a minimum 2-year follow-up. Am J Sports Med, Vol.43, PP.1598-1605,2015. 